\title{
DINÂMICA POPULACIONAL DE QUATRO ESPÉCIES DE DYSDERCUS (HEMIPTERA, PYRRHOCORIDAE) E A FENOLOGIA DAS PLANTAS HOSPEDEIRAS
}

\author{
J. R. de Almeida 1 \\ R. de Xerez ${ }^{2}$ \\ A. Caldas ${ }^{3}$
}

\begin{abstract}
POPULATIONAL DYNAMIC OF FOUR SPECIES OF DYSDERCUS (HEMIPTERA, PYRRHOCORIDAE) AND THE PHENOLOGY OF THE HOST PLANTS. The food cycle and geographical variation of Dysdercus diet were studied as strategy elements of trophic resources exploitation. The species used were D. fulvoniger, D. maurus, D. mendesi and D. ruficollis. A random sampling method was adopted at two regular sampling areas (Vassouras and Itaguaí, Rio de Janeiro, Brazil). The places sampled only once were selected based on the potential migration of Dydercus. Various transects were determined, with 99 places; these transects involved six morpho-climatic-phytogeographical domains: Atlantic Tropical, Cerrado, Caatinga, Amazonic Equatorial, Pantanal and "Planalto das Araucárias". The food cycle of Dysdercus at Vassouras is based on the exploitation of reproductive structures (flower and fruit) of some Malvaceae, Boraginaceae, Compositae and Cucurbitaceae. The total diet includes other host plant structures. Fifty-three plant species ( 16 families) are explored as food by the insect along the 99 places of sampling. The habitats of Dysdercus are characterized by climatic variations, geographical distribution and phenology of host plants, and also by the dispersion of seeds.
\end{abstract}

KEY WORDS. Hemiptera, Pyrrhocoridae, Dysdercus, populational dynamic, phenology, host plants

Espécies animais diferem de nichos ecológicos, entre outras causas, devido ao deslocamento de caracteres (BROWN \& WILSON, 1956), seleção de habitats (MACARTHUR, 1958; LANCIANI, 1970) ou por diferenças na dieta alimentar (SHAPIRO \& CARDÉ, 1970; PRICE, 1975).

Como os recursos de uma área são limitados, pode haver uma competição interespecífica. SHAPIRO \& CARDÉ (1970), GILBERT \& SINGER (1976) e BENSON (1978) relatam alguns casos de divisão de recursos alimentares entre

1) Departamento de Ecologia, Universidade Federal do Rio de Janeiro, Caixa Postal 68020, 21949-900 Rio de Janeiro, Rio de Janeiro, Brasil.

2) Instituto de Biologia, Universidade Federal Rural do Rio de Janeiro, Antiga Rodovia Rio-São Paulo KM 47, 23851-920 Itaguaí, Rio de Janeiro, Brasil.

3) Instituto de Biologia, Universidade Estadual do Rio de Janeiro, Rua Franscisco Xavier, 524 PHLC, sala 500, 20550-013 Rio de Janeiro, Rio de Janeiro, Brasil. 
lepidópteros. Assinalam que esta partição é conseguida de diferentes maneiras, tais como preferência alimentar por determinadas espécies de plantas hospedeiras, ocupação de diferentes habitats e também por separação dos fitófagos por estados fenológicos das plantas hospedeiras.

Neste trabalho estudaram-se os hábitos alimentares, mais especificamente a variação temporal da dieta de 4 espécies de Dysdercus em duas comunidades ruderais (Vassouras e Itaguaí, RJ), com o objetivo de conhecer a relação das variações de intensidades populacionais (Ip) destes percevejos com a fenologia das plantas hospedeiras. As espécies foram D. fulvoniger, D. maurus, D. mendesi e D. ruficollis.

\section{MATERIAL E MÉTODOS}

\section{Área de Estudo.}

Vassouras (RJ). Esta área faz parte da fazenda Oriente, localizada no município de Vassouras, Estado do Rio de Janeiro, próximo ao Km 18 da BR125 $\left(43^{\circ} 38^{\prime} \mathrm{W} / 22^{\circ} 25^{\prime} \mathrm{S}\right)$. A fazenda mede cerca de 100 ha e a atividade predominante é a pecuária. A área de estudo, com cerca de $6.200 \mathrm{~m}^{2}$, era usada para pasteio de bovinos. O solo é de textura média, argilo-arenoso e ácido. A distribuição de nutrientes não é uniforme, havendo carência nas elevações e excesso nas planíces. Esta área apresenta-se parcialmente inundada nos meses mais chuvosos (dezembro a abril), devido ao aumento do nível de água do córrego que a atravessa.

Os campos de pastagem correspondem à área primitivamente coberta por matas relativamente densas nas regiões elevadas e pos planícies pantanosas nas baixadas. A partir de 1944, a região começou a ser modificada com desmatamentos sucessivos, dragagem do rio e formação dos pastos. Da vegetação primitiva restam apenas núcleos, esparsamente distribuídos, de árvores.

O levantamento da vegetação constou de coletas de ramos com flores e frutos. O material coletado foi prensado e secado pelos procedimentos convencionais, e depositado no herbário da Universidade Federal Rural do Rio de Janeiro (RBR). A identificação das espécies foi feita pela Dra. Ariane Luna Peixoto (UFRURJ) e pelo Dr. M. Kaprovickas (Museo de La Plata, Argentina). A cobertura vegetal dos campos é herbácea, uniforme, com dominância de quatro espécies cultivadas de gramíneas: capim colonião (Panicum maximum Jacq.), capim elefante (Pennisetum purpureum Schum.), capim marmelada (Brachiaria plantaginea Link) e capim angola (Brachiaria purpurescens Henr.), todos inicialmente semeados.

Itaguaí (RJ). E uma área desmatada, localizada no município de Itaguaí, Estado do Rio de Janeiro, à margem da BR 465, km $45\left(43^{\circ} 45^{\prime} \mathrm{W} / 22^{\circ} 51^{\prime} \mathrm{S}\right)$. A área estudada, com $5.000 \mathrm{~m}^{2}$, compreende parte de um terreno usado esporadicamente como pasto. O solo é de textura média, argilo-arenoso, e a topografia é plana. A cobertura vegetal é rala e herbácea, com predominância de gramíneas das espécies Brachiaria purpurescens e B. plantaginae. Esparsamente, há núcleos 
com área aproximada de $80 \mathrm{~m}^{2}$, formados exclusivamente de Sidastrum micranthum (Malvaceae) com cerca de 100 a 200 exemplares cada um.

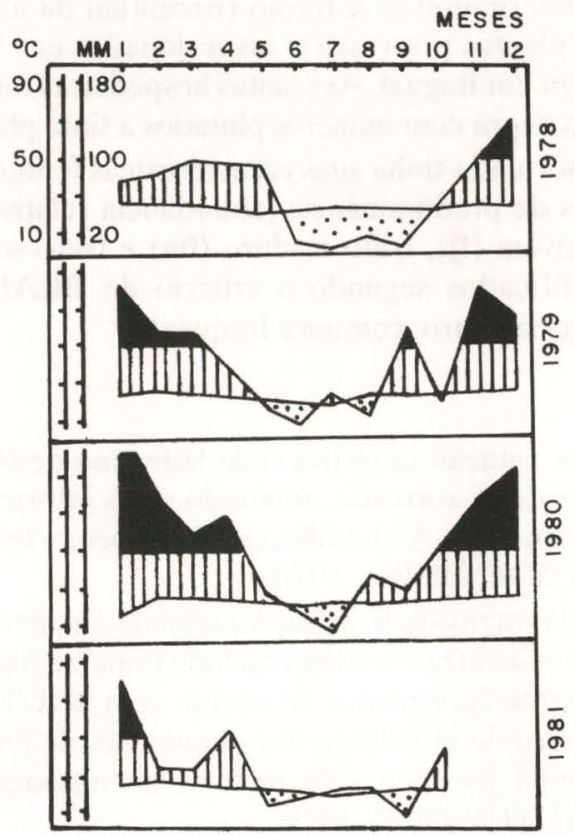

Fig. 1. Diagrama climático de Itaguaí-Vassouras (Rio de Janeiro) no período de 1978 a 1981 . As regiões pontilhadas representam períodos de seca; em linhas verticais, os períodos normais; em negrito, os período super-úmidos.

\section{Condições climáticas.}

Os dados meteorológicos foram obtidos na estação experimental de Itaguaí (PESAGRO, RJ, $43^{\circ} 41^{\prime} \mathrm{W} / 22^{\circ} 45^{\prime} \mathrm{S}$, altitude de $33 \mathrm{~m}$ ). $\mathrm{O}$ clima da região de Vassouras e Itaguaí, segundo classificação de Koppen, é tropical chuvoso. Pelo sistema Holdridge (PIANKA, 1978), ambos os locais encontram-se na zona de Floresta Úmida Tropical, e pela carta de vegetação de HUECK (1972) correspondem à Mata Tropical decídua e mesofítica com alta proporção de espécies sempre verdes.

Através do diagrama climático (Fig. 1), observa-se que na região estudada existe um ciclo anual basicamente constituído de um período super-úmido (outubro a abril) e um período de seca (junho e julho). A maior seca em intensidade e duração constatada no período de estudo foi em 1978, enquanto a maior precipitação, tanto em intensidade quanto em duração, foi registrada em 1980. 


\section{Amostragem.}

Amostras aleatórias simples foram colhidas mensalmente de abril de 1978 a março de 1981. Caso as plantas sorteadas tivessem sido eliminadas (por pastejo acidental ou doença), eram imediatamente substituídas por nova indicação aleatória. Determinou-se a fração percentual da amostra em $10 \%$, o que equivalia a dez plantas por espécie inspecionadas em Vassouras e 40 de Sidastrum micranthum em Itaguaí. As plantas hospedeiras foram marcadas por etiqueta de borracha negra com números pintados a tinta plástica branca.

Cada planta sorteada tinha suas características fenológicas registradas, através de anotações de predominância (abundância relativa) de botão floral (bf), flor (f), fruto jovem (fj), fruto maduro (fm) e fruto senescente (fs). Os fenons foram quantificados segundo o critério de BRAUN-BLANQUET (1950) em três categorias: raro, comum e frequente.

\section{Análise dos dados.}

Coletou-se por catação exaustiva cada indivíduo de Dysdercus presente em cada planta hospedeira sorteada, anotando-se as informaçôes pertinentes ao comportamento alimentar. A identificação das espécies foi feita com base no exame de genitálias (DOESBURG, 1968).

A estimativa da intensidade de exploração de Dysdercus por espécie de planta hospedeira foi feita através da intensidade populacional (Ip), que representa o número de insetos por planta, de acordo com SOUTHWOOD (1971). A estimativa do número de indivíduos das populações de Dysdercus nas áreas de Vassouras e Itaguaí foi feita pelo método da máxima verossimelhança (ZIPPIN, 1956 in SOUTHWOOD, 1971).

\section{Análise química.}

Para análise química $(\%)$ das estruturas reprodutivas de algumas plantas hospedeiras (Sidastrum micranthum, Sida rhombifolia, S. cordifolia) utilizou-se o aparelho de Soxlete e o método de Kjedahl (SCALES \& HARRINSON, 1920).

\section{RESULTADOS}

\section{Fenologia de Sidastrum micranthum}

Ocorrem anualmente dois picos de floração e frutificação. O primeiro em abril, com aproximadamente $70 \%$ das plantas em floração, tanto em Vassouras quanto em Itaguaí. Concomitantemente, a fase de frutificação atinge a média de $85,75 \%$ em Vassouras e $75 \%$ em Itaguaí (Figs 2 e 3). O segundo pico de floração dá-se em agosto, com média de 40,50\% de plantas em flor em ambas as áreas de estudo. Alguns frutos surgem em agosto; no entanto o pico de frutificação dá-se em setembro, atingindo 30,50\% em Itaguaí (Fig. 2) e 40,75\% em Vassouras (Fig. 3). 


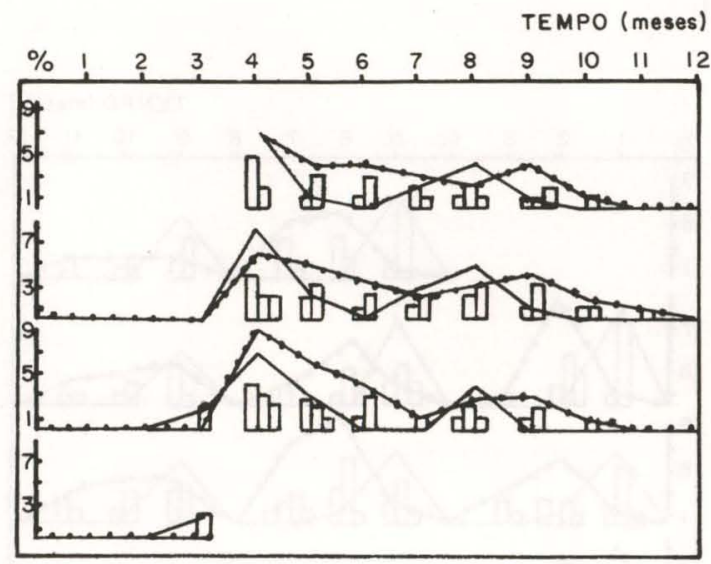

Fig. 2. Fenologia de Sidastrum micranthum em Itaguaí (RJ) no período de 1978 a 1981 (linha comum: floração; linha pontilhada: frutificação).

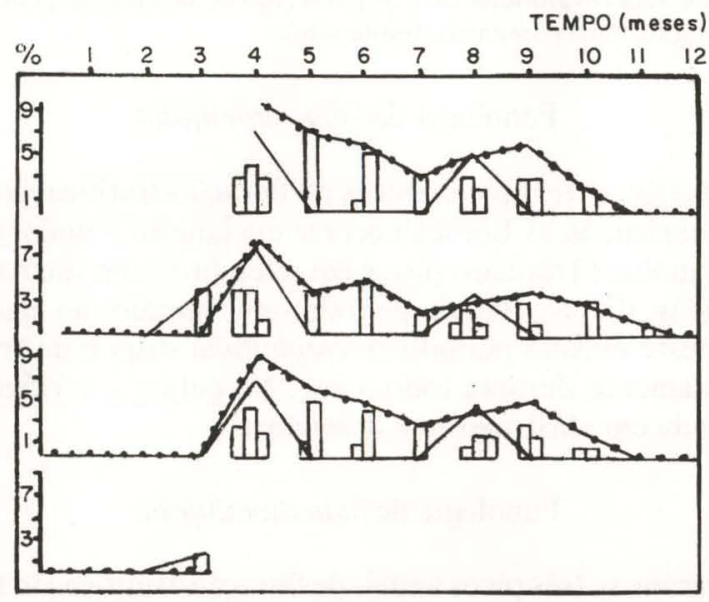

Fig. 3. Fenologia de Sidastrum micranthum em Vassouras (Rio de Janeiro) no período de 1978 a 1981 (linha comum: floração; linha pontilhada: frutificação).

A maior duração das estruturas reprodutivas nos exemplares de Itaguaí, em relação aos de Vassouras, provoca maior variação destas estruturas nas plantas em Itaguaí. As subfases da floração (botão floral e flor) são relativamente rápidas, enquanto as da frutificação são mais demoradas e assincrônicas. Como conseqüência há ocorrência de frutos em ambas as áreas desde abril até outubro (Figs 2 e 3 ).

As fenofases dos períodos super-úmidos atingem valores maiores que as dos períodos secos normais, i.e., o primeiro ciclo de floração e frutificação 
(período úmido), nos anos observados, sempre foi menor que o segundo ciclo (período seco).

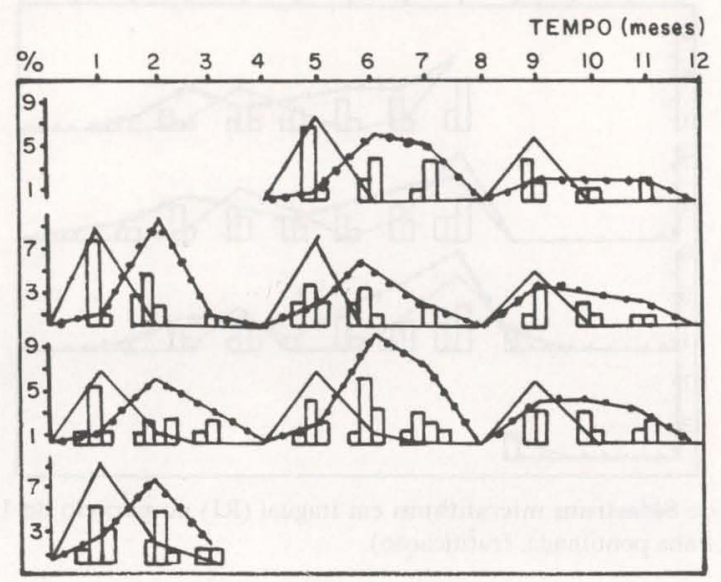

Fig. 4. Fenologia de Sida carpinifolia em Vassouras (Rio de Janeiro) no período de 1978 a 1981 (linha comum: floração; linha pontilhada: frutificação).

\section{Fenologia de Sida carpinifolia}

Constataram-se três picos anuais de floração-frutificação, sendo os dois primeiros assincrônicos. A floração ocorre em janeiro e maio, e a frutificação em fevereiro e junho. O terceiro pico é em setembro, com sincronia de floração e frutificação (Fig. 4). Os picos do período super-úmido são maiores que os do início e final deste mesmo período. S. carpinifolia dispõe de frutos nas várias subfases praticamente durante todo o ano. No entanto, verifica-se uma diminuição acentuada em abril, agosto e dezembro.

\section{Fenologia de Sida rhombifolia}

Observaram-se três picos anuais de floração-frutificação (excetuando-se março de 1980). Basicamente a floração se dá em março, junho e setembro, e a frutificação nos meses imediatamente seguintes (Fig. 5). Os picos de floraçãofrutificação de $S$. rhombifolia atingem valores ligeiramente inferiores àqueles de $S$. carpinifolia e $S$. micranthum.

As subfases botão floral e flor são efêmeras nas três espécies, mas fruto maduro e fruto senescente, principalmente este, têm longa duração.

Quanto às outras plantas hospedeiras, não se efetuaram observações quantitativas das fenofases de floração e frutificação. Após o sorteio, as várias espécies de plantas indicadas eram inspecionadas apenas sob o ponto de vista qualitativo. Por isso, as informações referem-se à identidade taxonômica e indicação da estrutura vegetal explorada como alimento pelas espécies de 
Dysdercus em estudo, bem como períodos e intensidades com que tais recursos eram usados.

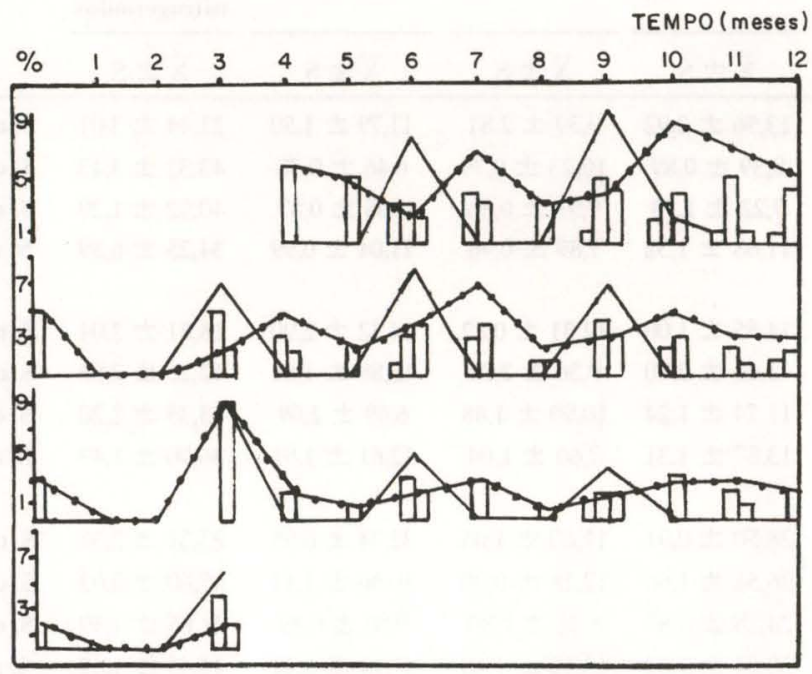

Fig. 5. Fenologia de Sida rhombifolia em Vassouras (Rio de Janeiro) no período de 1978 a 1981 (linha comum: floração; linha pontilhada: frutificação).

\section{Análise química}

A análise química (\%) das estruturas de algumas plantas hospedeiras de Vassouras estão na tabela I. O teor relativo (\%) de proteínas nessas plantas aumenta das fenofases botão floral-flor para fruto jovem, e decresce entre as fenofases fruto maduro para fruto senescente. Praticamente são insignificantes as diferenças intra e inter-específicas entre botão floral e flor; por outro lado, em fruto maduro e fruto jovem há algumas diferenças inter-específicas.

\section{Variação mensal da dieta}

Em Vassouras, não houve correlação positiva entre o número de diferentes espécies de plantas exploradas mensalmente por D. ruficollis, D. mendesi, D. maurus ou D. fulvoniger e o valor médio das intensidades de população (Ip) destas quatro espécies nas plantas hospedeiras respectivamente exploradas por elas. O coeficiednte de indeterminação $\left(1-r^{2}\right)$ foi alto para todos os casos, e a aplicação do teste $\mathbf{Z}$ para os valores de $\mathbf{r}$ (coeficiente de relação amostral) demonstrou não haver diferença significativa entre estes valores.

$\mathrm{Na}$ área de estudo em Itaguaí ocorreram $D$. maurus, $D$. fulvoniger, $D$. ruficollis e $D$. mendesi. A única hospedeira era $S$. micranthum, sendo todas as fenofases utilizadas por $D$. maurus e $D$. mendesi, enquanto as outras duas espécies só não utilizaram botão floral (Figs 2 e 6). 
Tabela I. Análise química (\%) das estruturas de algumas plantas hospedeiras de Vassouras, Rio de Janeiro.

\begin{tabular}{|c|c|c|c|c|c|}
\hline & Proteína & Mineral & Fibras & $\begin{array}{l}\text { Extratos não } \\
\text { nitrogenados }\end{array}$ & Espécie \\
\hline & $\bar{x} \pm s$ & $\bar{x} \pm s$ & $\bar{X} \pm s$ & $\bar{X} \pm s$ & \\
\hline & $13,56 \pm 2,92$ & $9,39 \pm 2,81$ & $11,79 \pm 1,50$ & $21,44 \pm 3,01$ & S. micranthum \\
\hline Botão & $8,39 \pm 0,89$ & $10,23 \pm 0,79$ & $6,46 \pm 0,78$ & $43,32 \pm 3,43$ & S. carpinifolia \\
\hline \multirow[t]{3}{*}{ Floral } & $7,22 \pm 1,73$ & $9,93 \pm 0,86$ & $4,05 \pm 0,87$ & $40,92 \pm 1,29$ & S. cordifolia \\
\hline & $11,68 \pm 1,32$ & $7,89 \pm 0,96$ & $11,04 \pm 0,99$ & $34,25 \pm 6,59$ & S. rhombifolia \\
\hline & $14,55 \pm 1,00$ & $12,31 \pm 0,82$ & $14,32 \pm 2,90$ & $18,81 \pm 2,04$ & S. micranthum \\
\hline \multirow[t]{4}{*}{ Flor } & $12,66 \pm 2,00$ & $9,36 \pm 2,04$ & $12,88 \pm 1,61$ & $42,26 \pm 2,83$ & S. carpinifolia \\
\hline & $11,74 \pm 1,24$ & $10,90 \pm 1,48$ & $6,69 \pm 2,09$ & $43,35 \pm 2,20$ & S. cordifolia \\
\hline & $13,87 \pm 1,31$ & $7,60 \pm 1,04$ & $12,61 \pm 1,91$ & $36,90 \pm 1,47$ & S. rhombifolia \\
\hline & $28,50 \pm 0,91$ & $11,60 \pm 1,05$ & $32,74 \pm 0,95$ & $22,31 \pm 2,31$ & S. micranthum \\
\hline Fruto & $26,51 \pm 1,66$ & $12,28 \pm 0,96$ & $10,46 \pm 1,11$ & $45,60 \pm 2,02$ & S. carpinifolia \\
\hline \multirow[t]{3}{*}{ Jovem } & $24,18 \pm 0,87$ & $8,26 \pm 0,80$ & $9,80 \pm 0,89$ & $41,65 \pm 1,59$ & S. cordifolia \\
\hline & $30,04 \pm 1,22$ & $13,52 \pm 1,48$ & $11,14 \pm 1,44$ & $43,45 \pm 1,69$ & S. rhombifolia \\
\hline & $10,38 \pm 0,85$ & $5,42 \pm 0,98$ & $24,28 \pm 0,57$ & $29,50 \pm 0,94$ & S. micranthum \\
\hline Fruto & $14,08 \pm 1,10$ & $10,10 \pm 1,39$ & $20,09 \pm 1,87$ & $46,94 \pm 1,05$ & S. carpinifolia \\
\hline \multirow[t]{3}{*}{ Maduro } & $11,81 \pm 1,17$ & $7,60 \pm 0,59$ & $12,10 \pm 0,98$ & $43,28 \pm 3,49$ & S. cordifolia \\
\hline & $19,22 \pm 1,62$ & $11,12 \pm 1,02$ & $21,33 \pm 1,34$ & $42,28 \pm 3,78$ & S. rhombifolia \\
\hline & $3,71 \pm 1,23$ & $3,42 \pm 1,01$ & $17,85 \pm 3,62$ & $32,49 \pm 2,66$ & S. micranthum \\
\hline Fruto & $10,26 \pm 1,91$ & $8,22 \pm 1,83$ & $34,45 \pm 2,51$ & $49,87 \pm 2,15$ & S. carpinifolia \\
\hline \multirow[t]{2}{*}{ Senescente } & $7,04 \pm 0,84$ & $6,84 \pm 0,84$ & $14,67 \pm 1,49$ & $38,44 \pm 4,31$ & S. cordifolia \\
\hline & $13,67 \pm 1,40$ & $7,66 \pm 1,30$ & $32,29 \pm 2,14$ & $44,60 \pm 2,27$ & S. rhombifolia \\
\hline
\end{tabular}

\section{Variação da dieta de Dysdercus maurus}

Constataram-se dois picos populacionais para D. maurus em 1978 (Fig. 7). No primeiro, em maio, foram exploradas quatro espécies de plantas hospedeiras, destacando-se $S$. micranthum e $S$. rhombifolia, com intensidades populacionais (Ip) respectivamente iguais a 5 e 2,8 . No segundo, em agosto, também quatro espécies vegetais foram exploradas, principalmente $S$. micranthum $(\mathrm{I} p=1,3)$ e Vernonia scorpioides $(\mathrm{I} p=1,2)$. Neste ano, D. maurus explorou uma (abril, dezembro), três (junho, julho, novembro), quatro (maio, agosto, outubro) e até cinco espécies vegetais (setembro). Nos picos populacionais, quatro espécies eram exploradas, e nos menores valores de Ip apenas 
uma espécie foi utilizada ( $S$. micranthum em abril e Wedelia paludosa em dezembro, Ip $=0,3$ ).

Em 1979 observaram-se praticamente dois picos populacionais: abril e outubro (Fig. 7). No primeiro foram exploradas $S$. micranthum $(\mathrm{Ip}=2,1)$ e $S$. rhombifolia $(\mathrm{Ip}=0,2)$ e no segundo $S$. micranthum $(\mathrm{Ip}=1,4), S$. carpinifolia $(\mathrm{Ip}=2,3), S$. rhombifolia $(\mathrm{Ip}=3,1)$ e W. paludosa $(\mathrm{Ip}=3,9)$. D. maurus explorou uma (maio, dezembro), duas (fevereiro, abril, junho, julho, novembro) e quatro espécies vegetais (janeiro, agosto, setembro, outubro).

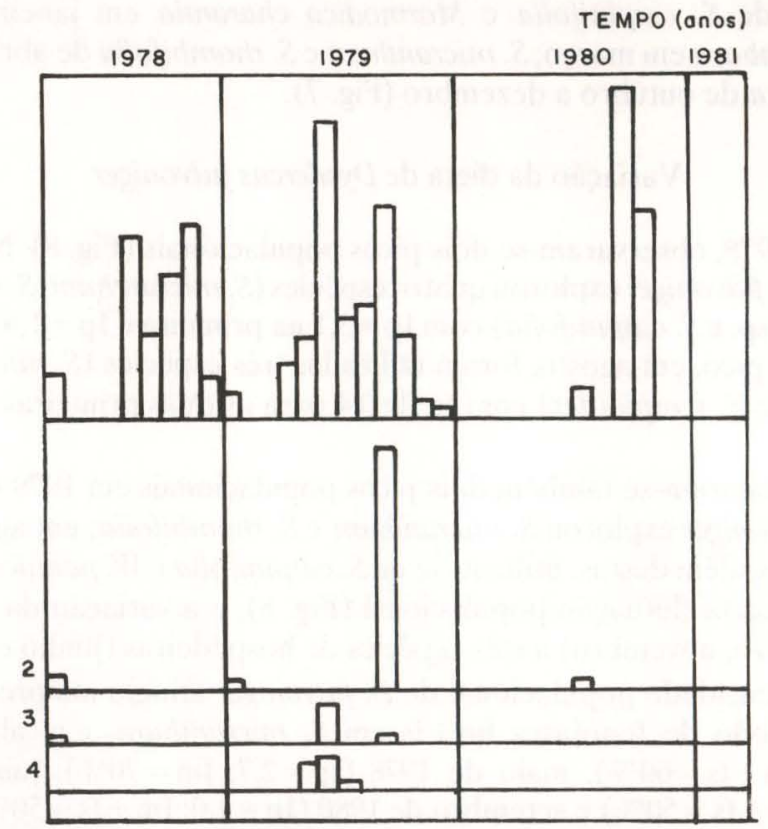

Fig. 6. Densidade mensal da população de Dysdercus spp. em Itaguaí (Rio de Janeiro). (1) D. maurus; (2) D. fulvoniger; (3) D. ruficollis; (4) D. mendesi.

Houve grande flutuação populacional de D. maurus em 1980, e a variação da dieta vegetal incluiu de uma (março, junho) a três espécies de hospedeiros (abril, maio, agosto, novembro).

Os maiores valores de Ip de $D$. maurus em $S$. micranthum tiveram relação principalmente com a fenofase de fruto maduro (fm) e fruto senescente (fs) (Figs 3 e 7); por exemplo, em maio de $1978(\mathrm{Ip}=5,7 ; \mathrm{fm}=70 \%)$, junho de 1978 $(\mathrm{Ip}=2,8 ; \mathrm{fs}=50 \%)$, setembro de $1979(\mathrm{Ip}=2,6 ; \mathrm{fm}+\mathrm{fs}=40 \%)$, junho de 1980 $(\mathrm{Ip}=4,8 ; \mathrm{fm}+\mathrm{fs}=50 \%)$, setembro de $1980(\mathrm{Ip}=3,6 ; \mathrm{fm}+\mathrm{fs}=40 \%)$ e outubro de $1980(\mathrm{Ip}=4,4 ; \mathrm{fs}=30 \%)$.

D. maurus também explora S. rhombifolia de modo similar (Fig. 7). Os valores de Ip são inferiores àqueles encontrados em $S$. micranthum, mas o períodọ de uso é basicamente o mesmo, e o aumento de Ip se dá nas fenofases 
de fruto maduro e fruto senescente $(\mathrm{fm}+\mathrm{fs})$, e.g. maio de $1978(\mathrm{Ip}=2,8$; $\mathrm{fs}=50 \%)$ e outubro de $1979(\mathrm{Ip}=3,1 ; \mathrm{fm}=50 \%)$.

A exploração de $D$. maurus em $S$. carpinifolia normalmente é baixa, exceto em setembro de 1979 e julho de 1980, em que houve aumento na Ip coincidente com as fenofases $\mathrm{fm}+\mathrm{fs}$; no restante do período, o nível de Ip foi praticamente homogêneo (Fig. 4). Basicamente as fenofases desta hospedeira utilizadas pelos Dysdercus foram as reprodutivas. As demais hospedeiras, afora $S$. carpinifolia, foram exploradas apenas na fenofase flor.

O ciclo alimentar de D. maurus em Vassouras é caracterizado por exploração de S. carpinifolia e Mormodica charantia em janeiro-fevereiro; Cordia corymbosa em março; S. micranthum e $S$. rhombifolia de abril a outubro; e $W$. paludosa de outubro a dezembro (Fig. 7).

\section{Variação da dieta de Dysdercus fulvoniger}

Em 1978, observaram-se dois picos populacionais (Fig. 8). No primeiro, em junho, D. fulvoniger explorou quatro espécies (S. micranthum, S. rhombifolia, Eupatorium sp. e $S$. carpinifolia) com Ip $=3,1$ na primeira e Ip $=1,4$ na segunda. No segundo pico, em agosto, foram utilizadas três espécies (S. micranthum, $S$. rhombifolia e V. scorpioides) com Ip de 0,4 para as duas primeiras e 0,3 para a última.

Constataram-se também dois picos populacionais em 1979 (Fig. 8). Em abril, D. fulvoniger explorou S. micranthum e S. rhombifolia; em agosto idem e em setembro, além destas, utilizou-se de $S$. carpinifolia e W. paludosa. Em 1980 houve uma certa flutuação populacional (Fig. 8), e a variação da dieta foi de uma (fevereiro, novembro) a três espécies de hospedeiras (junho e setembro).

A intensidade populacional de $D$. fulvoniger atingiu maiores valores no mesmo período de fenofases $\mathrm{fm}+\mathrm{fs}$ em S. micranthum, e.g. abril de 1978 $(\mathrm{Ip}=3,1 ; \mathrm{fm}+\mathrm{fs}=60 \%)$, maio de $1978(\mathrm{Ip}=2,7 ; \mathrm{fm}=70 \%)$, junho de 1980 $(\mathrm{Ip}=2,4 ; \mathrm{fm}+\mathrm{fs}=50 \%)$ e setembro de $1980(\mathrm{Ip}=1,0 ; \mathrm{fm}+\mathrm{fs}=50 \%)$.

$S$. rhombifolia foi explorada por $D$. fulvoniger em menor intensidade que S. micranthum (Fig. 8). Todas as fenofases reprodutivas da hospedeira foram utilizadas, destacando-se botão floral e flor (bf $+f$ ) em junho de 1979 , onde o $\mathrm{I} p=1,4 \mathrm{e}$ bf $+\mathrm{f}=60 \%$ (Figs $5 \mathrm{e} 8$ ). A utilização de $S$. carpinifolia por $D$. fulvoniger verifica-se principalmente no fruto maduro e fruto senescente (Figs 4 e 8 ).

O ciclo alimentar de D. fulvoniger em Vassouras caracteriza-se por utilização de $S$. carpinifolia em fevereiro e outubro, $S$. micranthum e $S$. rhombifolia de abril a agosto, e esta última em novembro.

\section{Variação da dieta de Dysdercus ruficollius}

Ocorreram três picos populacionais de D. ruficollis em 1978 (Fig. 9). No primeiro foram exploradas duas espécies hospedeiras, $S$. micranthum e $S$. rhombifolia ( $\mathrm{Ip}=4,4$ e 3,2 respectivamente). No segundo, foram utilizadas $S$. micranthum $(\mathrm{Ip}=1,3), S$. rhombifolia $(\mathrm{I} \mathrm{p}=1,1), C$. corymbosa e $V$. scorpioides 


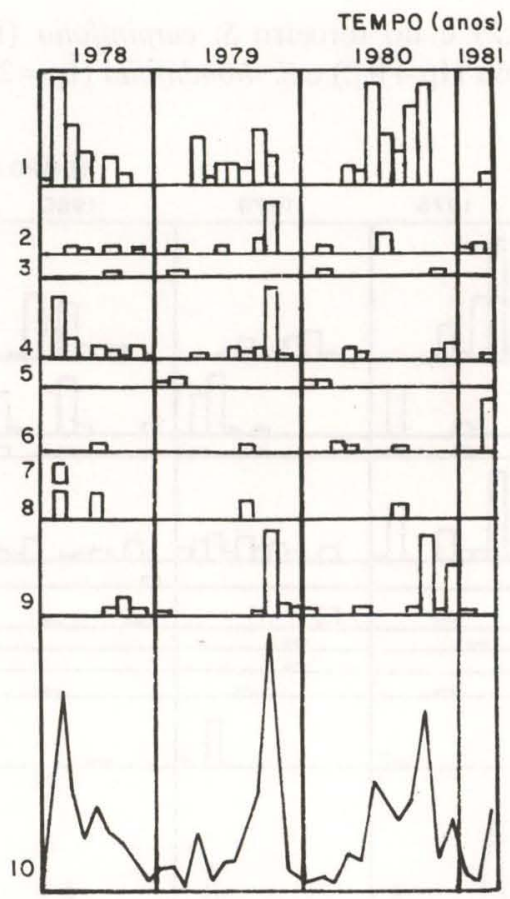

Fig. 7. Densidade mensal de D. maurus em Vassouras (Rio de Janeiro) estimada por Intensidade Populacional e método da Máxima Verossimelhança. (1) S. micranthum; (2) S. carpinifolia; (3) S. cordifolia; (4) S. rhombifolia; (5) M. charantia; (6) S. corimbosa; (7) Eupatorium sp.; (8)V. scorpioides; (9) W. paludosa; (10) Estimativa da densidade de D. maurus na área de coleta.

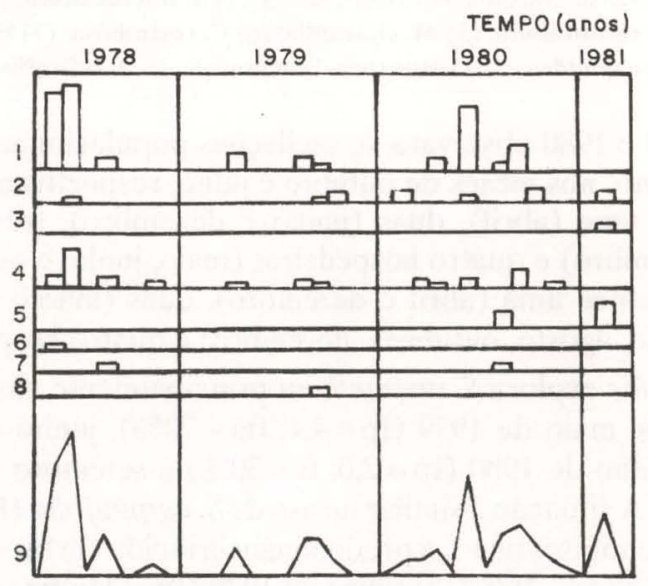

Fig. 8. Densidade mensal de D. fulvoniger em Vassouras (Rio de Janeiro) estimada por Intensidade Populacional e método da Máxima Verossimelhança. (1) S. micranthum; (2) S. carpinifolia; (3) S. cordifolia; (4) S. rhombifolia; (5) C. corimbosa; (6) Eupatorium sp.; (7) V. scorpioides; (8) W. paludosa; (9) Estimativa da densidade de D. fulvoniger na área de coleta. 
(ambas com $\mathrm{Ip}=0,2)$ e no terceiro $S$. carpinifolia $(\mathrm{Ip}=2,8), S$. cordifolia $(\mathrm{Ip}=0,2), W$. paludosa $(\mathrm{I} \mathrm{p}=0,2)$ e $S$. rhombifolia $(\mathrm{I} \mathrm{p}=2,0)$.

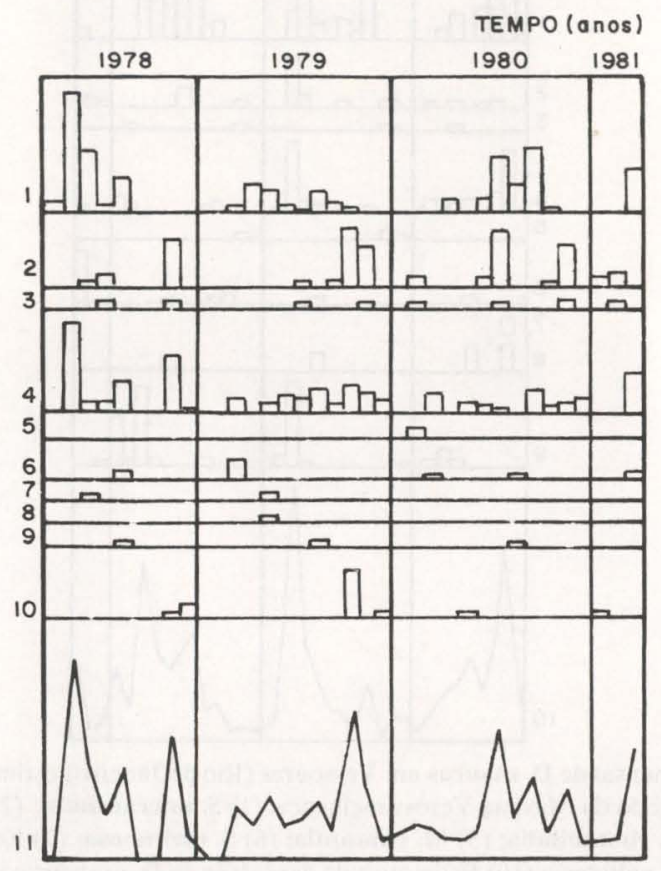

Fig. 9. Densidade mensal de D. ruficollis em Vassouras (Rio de Janeiro) efetuada por Intensidade Populacional e método da Máxima Verossimelhança. (1) S. micranthum; (2) S. carpinifolia; (3) S. cordifolia; (4) S. rhombifolia; (5) M. charantia; (6) C. corimbosa; (7) Eupatorium sp.; (8) V. miersiana; (9) V. scorpioides; (10) Estimativa da densidade de D. ruficollis na área de coleta.

Em 1979 e 1980 observara-se oscilações populacionais (Fig. 9) com um pico em cada ano, nos meses de outubro e julho, respectivamente. A dieta em 1979 englobou uma (abril), duas (junho e dezembro), três (março, agosto, setembro, novembro) e quatro hospedeiras (maio, junho e outubro). Em 1980, a dieta variou entre uma (abril e dezembro), duas (março e setembro), três (fevereiro, junho, agosto, outubro e novembro) e quatro hospedeiras (julho).

D. ruficollis explora $S$. micranthum principalmente nas fenofases $\mathrm{fm}+\mathrm{fs}$ (Figs 3 e 9), e.g. maio de 1979 ( $\operatorname{Ip}=4,4 ; \mathrm{fm}=70 \%)$, junho de $1979(\operatorname{Ip}=2,2$; $\mathrm{f}+\mathrm{fs}=60 \%)$, julho de $1980(\mathrm{Ip}=2,0 ; \mathrm{fs}=30 \%)$ e setembro de $1980(\mathrm{Ip}=2,3$; $\mathrm{fm}+\mathrm{fs}=50 \%$ ). A situação é similar no uso de S. carpinifolia (Figs 4 e 9). Apesar de explorar as fenofases $\mathrm{bf}+\mathrm{f}$, a predominância incide $\mathrm{em} \mathrm{fm}+\mathrm{fs}$, e.g. novembro de $1978(\mathrm{Ip}=1,8 ; \mathrm{fs}=20 \%)$, outubro de $1979(\mathrm{Ip}=2,0 ; \mathrm{fm}=30 \%)$, novembro de $1979(\mathrm{Ip}=1,4 ; \mathrm{fm}+\mathrm{fs}=20 \%)$ e julho de $1981 \mathrm{Ip}=2,0 ; \mathrm{fm}+\mathrm{fs}=60 \%)$.

Em S. rhombifolia, D. ruficollis utiliza também botão floral (bf) e flor (f), mas os maiores valores de Ip estão principalmente nos meses de fenofases de 
$\mathrm{fm}+\mathrm{fs}$ (Figs 5 e 9), e.g. maio de $1978(\mathrm{Ip}=3,2 ; \mathrm{fs}=60 \%)$ e novembro de 1978 $(\mathrm{Ip}=2,0 ; \mathrm{fm}+\mathrm{fs}=70 \%)$. Excetuando-se $S$. cordifolia , as demais hospedeiras em Vassouras são utilizadas apenas na fenofase flor.

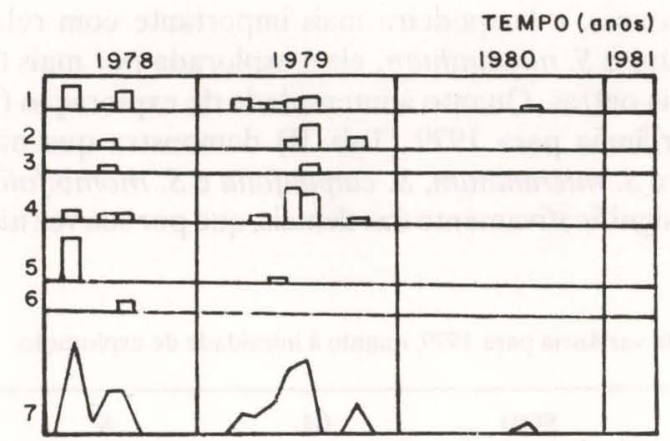

Fig. 10. Densidade mensal de D. mendesi em Vassouras (Rio de Janeiro) efetuada por Intensidade Populacional e método da Máxima Verossimelhança. (1) S. micranthum; (2) S. carpinifolia; (3) S. cordifolia; (4) S. rhombifolia; (5) V. miersiana; (6) V. scorpiodes. (7) Estimativa da densidade de D. mendesi na área de coleta.

\section{Variação da dieta de Dysdercus mendesi}

Nos anos de 1978 e 1979 observaram-se dois picos populacionais em cada ano. Em 1980 apenas uma ocorrência foi registrada (em S. micranthum) na área de estudo (Fig. 10). No primeiro pico de 1978 a dieta constou de $S$. micranthum $(\mathrm{Ip}=0,8), S$. rhombifolia $(\mathrm{I} p=0,2)$ e Vernonia miersiana $(\mathrm{I} \mathrm{p}=1,6)$; no segundo, incluiu $S$. micranthum (Ip = 0,2 em julho e $0,7 \mathrm{em}$ agosto), $S$. carpinifolia $(\mathrm{Ip}=0,4$ em julho), $S$. cordifolia (Ip =0,2 em julho), $S$. rhombifolia $(\mathrm{Ip}=0,2$ em julho e agosto) e $V$. scorpioides (Ip = 0,2 em agosto). Em 1979, no primeiro pico, a dieta constou de $S$. micranthum (Ip $=0,4$ em junho e julho), $S$. carpinifolia $(I p=0,3$ em julho e 0,8 em agosto) e $S$. rhombifolia (Ip =1,2 em junho e 0,9 em julho). No segundo pico, $D$. mendesi fez uso de $S$. carpinifolia $(\mathrm{Ip}=0,4)$ e $S$. rhombifolia $(\mathrm{Ip}=-0,1)$.

Os valores de Ip de D. mendesi por espécie de planta hospedeira são baixos (Fig. 10). Exploram todas as fenofases em $S$. micranthum e $S$. rhombifolia (Figs 3, 5 e 10), excluem apenas a fenofase botão floral (bf) em $S$. carpinifolia (Figs 4 e 10) e nas demais hospedeiras utilizam apenas a fenofase flor (f).

\section{Dieta das demais espécies de Dysdercus}

$\mathrm{Na}$ área de estudo em Vassouras, de abril de 1978 a março de 1981, foram encontradas outras espécies de Dysdercus. Entre estas, D. honestus explorando S. micranthum (maio de 1978, Ip =3,2; junho de 1978, Ip =1,2 e agosto de 1978, 1979 e 1980, todos com Ip=0,4), C. corymbosa (agosto de 1978 e 1979 com I $=1,0$ e 0,4 respectivamente) e $V$. scorpioides (agosto de 1978, 1979 e 1980, 
com Ip $=1,2,0,4$ e 0,2 respectivamente). Também $D$. immarginatus em $S$. carpinifolia e D. obscuratus em S. rhombifolia.

\section{DISCUSSÃO}

Em Vassouras, a hospedeira mais importante com relação a tempo e intensidade de uso é $S$. micranthum, ela é explorada por mais tempo (duração em meses) que as outras. Quanto à intensidade de exploração (baseada no Ip), a análise de variância para 1979 (Tab. II) demonstra que não há diferença significante entre $S$. micranthum, $S$. carpinifolia e $S$. rhombifolia, mas que este conjunto difere significativamente das demais, que por sua vez não diferem entre si.

Tabela II. Análise de variância para 1979, quanto à intesidade de exploração.

\begin{tabular}{lrrrr}
\hline & SQD & GL & \multicolumn{1}{c}{ S $^{2}$} & F \\
\hline Variação total & 11225,992 & 119 & & \\
Variação entre plantas & 4538,083 & 9 & 504,231 & $18,482 *$ \\
Variação entre meses & 3987,100 & 11 & 362,463 & $13,286^{*}$ \\
Erro de amostragem & 2700,808 & 99 & 27,280 & \\
\hline
\end{tabular}

*. Estatisticamente significativo para $\mathrm{P}=0,95$

Durante o período de estudo, observou-se que os picos populacionais e os maiores valores de Ip de $D$. maurus acompanharam as fenofases fruto maduro (fm) e fruto senescente (fs) em S. micranthum e S. rhombifolia, em intensidade de exploração e tempo de uso. Estas hospedeiras devem ser fundamentais para a manutenção de $D$. maurus nesta comunidade. Ocasionalmente $D$. maurus explorou W. paludosa, atingindo elevado Ip apenas em outubro de 1979 e 1980. As demais hospedeiras foram exploradas numa intensidade diminuta e normalmente de um a três meses, enquanto Eupatorium sp. só foi explorada uma vez.

D. fulvoniger também teve picos populacionais durante as fenofases $\mathrm{fm} \mathrm{e}$ fs de $S$. micranthum. A utilização de $S$. carpinifolia verifica-se principalmente nas fenofases $\mathrm{fm}$ e fs, enquanto em $S$. rhombifolia dá-se em bf $+\mathrm{f}$. Regularmente são utilizadas $S$. carpinifolia e $V$. scorpioides, e esporadicamente as demais. A dieta de $D$. ruficollis envolve principalmenmte as fenofases $\mathrm{fm}$ e $\mathrm{fs}$ de $S$. micranthum, $S$. carpinifolia e $S$. rhombifolia. Praticamente há uma alternância temporal na exploração das duas primeiras hospedeiras. Os dois primeiros picos populacionais do ano têm relação com exploração de $S$. micranthum e os outros dois com $S$. carpinifolia; $S$. rhombifolia é explorada com uma taxa menor por maior período de tempo. A exploração de C. corymbosa, $V$. scorpioides, $S$. cordifolia e Eupatorium sp. é mínima, mas regular, enquanto a de $M$. charantia e V. miersiana é esporádica.

A maior incidência da exploração nas fenofases $\mathrm{fm}+\mathrm{fs}$ pode ser parcialmente justificada pela qualidade química do recurso explorado. Segundo 
CARTIER (1968) as sementes geralmente têm alto teor de vitaminas do complexo B, amido, ferro e proteínas. Nas sementes em que a concentração de proteínas é baixa, esta é compensada pela diversidade de proteínas dentro da semente (CARTIER, 1968). Como as sementes de $S$. micranthum, S. cordifolia e $S$. carpinifolia têm baixo teor relativo de proteína, e como estas plantas constituem a dieta principal das espécies de Dysdercus estudadas, é possível que estas plantas tenham elevada diversidade proteica nas sementes. Ainda CARTIER observou que os insetos discriminam bem variações na dieta, e assinala que os aminoácidos e sacarose, além do $\mathrm{pH}$, regulam o comportamento alimentar de muitas espécies, funcionando como estímulo nutritivo. EDWARDS \& WRATTEN (1981) ao contrário, afirmam: "os nutrientes devem ter uma papel, mas nenhum inseto é totalmente polífago e as necessidades de nutrientes da maioria deles é semelhante; assim, é difícil ver como somente a concentração de nutrientes pode explicar a seleção específica ou rejeição da espécie de planta". KENNEDY (1968) propõe a teoria da "discriminação dualística", segundo a qual a seleção das plantas hospedeiras é baseada em um estímulo produzido pelas substâncias secundárias e outro pelos nutrientes, ou seja, o balanço da intensidade de preferência de um inseto entre nutrientes e sabor será relacionado com a posição do inseto no espectro monofagia/polifagia.

Quanto às estratégias de exploração dos recursos tróficos, dois parâmetros são fundamentais: intensidade e duração total de exploração. Para analisá-los, considerou-se a Ip como estimativa de intensidade de exploração e a duração em meses para medir o tempo de exploração.

Considerando-se uma hipótese que envolve duas estratégias: a minimização de tempo (min.t) e maximização de exploração (max. e), e sua recíproca maximização do tempo (max. t.) e minimização de exploração (min. e.); as espécies de Dysdercus que exibem a primeira estratégia são encontradas sobre a mesma espécie de hospedeira, de um a dois meses isoladas dos outros meses do mesmo ano e com uma Ip maior que dois adultos/planta. Para os Dysdercus que exibem a segunda estratégia, o tempo em que são encontrados sobre uma mesma espécie de hospedeira é igual ou superior a três meses, e têm uma Ip maior que dois adultos/planta.

Na primeira estratégia há uso dos recursos até o limite de não interferir com a sequência fenológica da estrutura vegetal explorada. Na segunda há exploração de poucos recursos, até atingir o nível extremo de afetar o desenvolvimento fenológico da planta. No entanto, a exploração seria numa quantidade que permitisse o clímax de um razoável número de recursos explorados. Evidentemente as duas estratégias são mutuamente exclusivas, sendo inclusive possível arranjos entre seus aspectos fundamentais. Dentro desse contexto, as populações de Dysdercus usam flexivelmente parte do repertório compreendido entre as duas estratégias de exploração.

De acordo com aquela hipótese relativa à dieta e esta pertinente à estratégia de exploração, observou-se que em Vassouras a dieta principal de $D$. ruficollis era constituída de $S$. micranthum e $S$. carpinifolia, utilizadas com max.t. 
e max.e., e $S$. rhombifolia com max.t. e min.e. mas, inicialmente (maio a novembro de 1978) também com max.e. A dieta complementar constava de $S$. cordifolia, C. corymbosa, Eupatorium sp., V. scorpioides e W. paludosa, e a dieta esporádica de $M$. charantia e $V$. miersiana. Em Itaguaí, $D$. ruficollis explorava $S$. micranthum com max.e. e min.t.

A dieta principal de $D$. maurus incluía $S$. micranthum explorada com max.e e min.t., tanto em Vassouras como em Itaguaí. A dieta principal comportava ainda, em Vassouras, S. rhombifolia explorada com max.t. e min.e. e esporadicamente com max.e.; $W$. paludosa com max.t. e max.e e $S$. carpinifolia com max.t. e min.e. A dieta complementar constava de $S$. cordifolia, $M$. charantia, C. corymbosa e V. scorpioides, e a esporádica apenas de Eupatorium.

Para $D$. fulvoniger a dieta principal era formada por $S$. micranthum explorada com max.t e max.e. em Vassouras, e max.t. e min.e. em Itaguaí. Em Vassouras, a dieta incluía também S. rhombifolia usada com max.t. e min.e. Na dieta complementar estavam $S$. carpinifolia e $V$. scorpioides, enquanto $W$. paludosa, Eupatorium sp., $S$. cordifolia e $C$. corymbosa pertenciam à dieta esporádica.

D. maurus, $D$. fulvoniger e $D$. ruficollis exploram $S$. micranthum e $S$. rhombifolia em comum na dieta principal. D. maurus e D. ruficollis exploravam, além destas, $S$. carpinifolia. S. micranthum e $S$. rhombifolia eram exploradas através dos mesmos tipos de estratégias pelas três espécies, respectivamente: max.e. e min.t., max.e. e max.t., e max.e. e min.t. No entanto, $S$. carpinifolia era explorada por estratégias diferentes por $D$. ruficollis (max.e. e max.t.) e $D$. maurus (min.e. e max.t.); D. maurus explorava W. paludosa como componente da dieta principal, enquanto $D$. ruficollis a incluía na dieta complementar (min.t. e min.e.) e $D$. fulvoniger na dieta esporádica.

No elenco das hospedeiras secundárias, $S$. cordifolia e $C$. corymbosa são comuns a $D$. maurus e $D$. ruficollis. Entre os três meses de exploração destas duas espécies, $S$. cordifolia é usada comumente só em fevereiro, enquanto $C$. corymbosa o é em março e agosto.

$V$. scorpioides pertenceria à dieta secundária de D. maurus, D. ruficollis e $D$. fulvoniger, sendo explorada em comum pelas referidas espécies no mês de agosto. V. miersiana somente é explorada por D. ruficollis, e mesmo assim só na dieta esporádica.

Entre as três espécies constantes em Vassouras, D. maurus parece ser a mais eclética na dieta principal, $D$. ruficollis na dieta complementar e $D$. fulvoniger na dieta esporádica. Isto tem reflexo na Ip e picos populacionais. $D$. maurus apresenta os maiores valores de Ip e picos populacionais, seguido de $D$. ruficollis e $D$. fulvoniger.

Comparando-se a flutuação das populações de D. maurus, D. ruficollis e D. fulvoniger, nota-se entre elas algumas características de semelhança. $\mathrm{O}$ número de picos populacionais por ano nas três espécies varia de três a quatro, levando a crer que haja igual quantidade de gerações anuais. Apesar de os picos populacionais de $D$. maurus serem maiores que os outros e de haver uma 
variação nos níveis populacionais superiores e inferiores de um ano para outro, a dinâmica em cada ano de estudo foi muito parecida. Isto sugere que as três espécies são afetadas de modo semelhante por certos fatores ecológicos.

Observou-se que os maiores picos populacionais das três espécies estão quase numa mesma faixa de tempo, enquanto os picos menores têm dissociações temporais. Os maiores níveis populacionais são atingidos na fase considerada climaticamente normal. Durante os períodos secos e super-úmidos há diminuição numérica nas populações. Parece que estas espécies de Dysdercus são mais afetadas pelas chuvas que pelas secas. Provavelmente o encharcamento do solo é mais prejudicial aos ovos e ninfas de primeiro estádio que a evapotranspiração. A vegetação ruderal é capaz de reter certa umidade, mas não dá vazão à absorção do excesso de volume d'água proveniente da cheia e do transbordamento do córrego.

A fenologia das plantas hospedeiras também é afetada pelo regime climático, principalmente pela pluviosidade. Portanto, o ajuste de períodos reprodutivos dos Dysdercus com a fenologia de suas hospedeiras também está relacionado com as condições climáticas. Não há informações sobre o tamanho populacional das plantas hospedeiras em Vassouras, mas a densidade conjunta confere uma certa abundância, apesar de qualitativamente insatisfatória, pelo menos durante um certo período do ano.

Para MACARTHUR \& WILSON (1967) o tamanho máximo que uma população pode atingir depende da capacidade de suporte do ambiente (k). Afirmam que normalmente o número de indivíduos nas populações tropicais fica próximo de exaurir os recursos que um habitat pode suportar. Hipotetiza-se neste estudo que os principais componentes que determinam o nível "k" para as populações de Dysdercus são: (1) tamanho populacional e relativa assincronia fenológica do elenco de plantas hospedeiras para cada espécie de Dysdercus; (2) condições ambientais apropriadas, em termos de clima e solo, para colonização; (3) diversidade na dieta secundária e esporádica, incluindo aqui as possibilidades de predação. Possivelmente, não deve ser comum as populações de Dysdercus situarem-se próximo ao limite, porque as densidades populacionais são baixas, e cada planta poderia suportar um maior número de indivíduos. Além disso, a influência de Dysdercus sobre as hospedeiras ruderais parece ser mínima na área de estudo.

Não foi possível determinar a importância das populações de cada espécie de planta hospedeira, porque não se considerou o tamanho populacional nem a distribuição local de cada hospedeira. São necessários estudos mais amplos, envolvendo a dinâmica das populações de Dysdercus e das respectivas hospedeiras, assim como de toda a guilda de animais que exploram essas plantas.

A partir dos resultados discutidos, sugere-se que a divisão de recursos alimentares entre as espécies de Dysdercus é obtida por ecletismo alimentar, baixa densidade populacional, relativa divergência nos níveis de exploração de recursos comuns, diferenças na preferência alimentar e flexibilidade nas estratégias de exploração dos recursos altamente nutritivos. 


\section{REFERÊNCIAS BIBLIOGRÁFICAS}

BENSON, W.W. 1978. Resource partitioning in passion vine butterflies. Evolution, 32(3): 493-518.

BRAUN-BLANQUET, J. 1950. Sociologia vegetal. Buenos Aires, Acme Agency, 421p.

BROWN JR., W.L. \& E.O WILSON. 1956. Character displacement. Syst. Zool. 5: $49-64$.

CARTIER, J.J. 1968. Factors of host plant specificity and artificial diets. Bull. Entomol. Soc. Amer. 14 (1): 18-21.

DOESBURG JR., P.H. 1968. A revision of the New World species of Dysdercus Guérin Menéville (Heteroptera, Pyrrhocoridae). Heiden, Zool. Verhandel., 218p.

EDWARDS, F.J. \& S.D. WRANTTEM. 1981. Ecologia das interaçòes entre insetos e plantas. São Paulo, EDUSP, 71p.

GILBERT, L.E. \& M.C. SINGER. 1976. Butterfly ecology. Ann. Rev. Ecol. Syst. 6: 365-97.

HUECK, K. 1972. As florestas da América do Sul. Rio de Janeiro, Nacional, $466 \mathrm{p}$.

KENNEDY, J.S. 1968. Phuysiological condition of the host plant and susceptibility to aphid attack. Bull. Entomol. Soc. Amer. 14 (1): 18-21.

LANCIANI, C.A. 1970. Resource partitioning in species of the mite gebus Eylais. Ecology 51: 338-342.

MACARTHUR, R.M. 1958. Population ecology of some warblers of northeastern coniferous forest. Ecology 39: 599-619.

MACARTHUR, R.M. \& E.O. WILSON. 1967. The theory of island biogeography. New Jersey, Princeton, 203p.

PIANKA, E.R. 1978. Evolutionary ecology. New York, Harper \& Row, 397p.

PRICE, D.W. 1975. Insect ecology. New York, Wiley, 514p.

SCALES, F.M. \& A.P. HARRISON. 1920. Boric acid modification of the Kjeldah method for crop and soil analysis. J. Ind. Eng. Chem. 12: 350.

SHAPIRO, A.M. \& R.T. CARDÉ. 1970. Habitat selection and competition among sibling species of satyrid butterflies. Evolution 24: 48-54.

SOUTHWOOD, T.R.E. 1971. Ecological methods. With particular reference to the study of insect populations. London, Chapman \& Hall, 391p.

Recebido em 05.IV.1991; aceito em 25.XI.1994. 